\title{
Use of GC-MS based metabolic fingerprinting for fast exploration of fungicide modes of action
}

\author{
Zhihong Hu, Tan Dai, Lei Li, Pengfei Liu* and Xili Liu
}

\begin{abstract}
Background: The widespread occurrence of fungicide resistance in fungal plant pathogens requires the development of new compounds with different mode(s) of action (MOA) to avoid cross resistance. This will require a rapid method to identify MOAs.

Results: Here, gas chromatography-mass spectrometry (GC-MS) based metabolic fingerprinting was used to elucidate the MOAs of fungicides. Botrytis cinerea, an important pathogen of vegetables and flowers, can be inhibited by a wide range of chemical fungicides with different MOAs. A sensitive strain of $B$. cinerea was exposed to $\mathrm{EC}_{50}$ concentrations of 13 fungicides with different known MOAs and one with unknown MOA. The mycelial extracts were analyzed for their "metabolic fingerprint" using GC-MS. A comparison among the GC-MS vector' profiles of cultures treated with fungicides were performeded. A model based on hierarchical clustering was established which allowed these antifungal compounds to be distinguished and classified coinciding with their MOAs. Thus, metabolic fingerprinting represents a rapid, convenient, and information-rich method for classifying the MOAs of antifungal substances. The biomarkers of fungicide MOAs were also established by an analysis of variance and included succinate for succinate dehydrogenase inhibitors and cystathionine for methionine synthesis inhibitors. Using the metabolic model and the common perturbation of metabolites, the new fungicide SYP-14288 was identified as having the same MOA as fluazinam.
\end{abstract}

Conclusion: This study provides a comprehensive database of the metabolic perturbations of $B$. cinerea induced by diverse MOA inhibitors and highlights the utility of metabolic fingerprinting for defining MOAs, which will assist in the development and optimization of new fungicides.

Keywords: Metabolic fingerprinting, GC-MS, Botrytis cinerea, Fungicide, Mode of action

\section{Background}

The fungal pathogen Botrytis cinerea causes serious losses in more than 200 crops worldwide. It can survive for relatively short periods as mycelia and/or conidia and for extended periods as sclerotia in crop debris [1]. The fungus causes grey mold disease, which can be controlled by the application of a wide range of chemical fungicides that act as seven modes of action (MOAs), including $\beta$-tubulin assembly inhibitors, respiration inhibitors, uncouplers of oxidative phosphorylation, methionine biosynthesis inhibitors, signal transduction inhibitors, sterol biosynthesis inhibitors,

\footnotetext{
* Correspondence: pengfeiliu@cau.edu.cn

Department of Plant Pathology, China Agricultural University, Beijing 100193, People's Republic of China
}

and multi-site inhibitors. Unfortunately, $B$. cinerea has developed high levels of resistance to most of the fungicides used for its control in the field [2-5]. Although many new fungicides that target $B$. cinerea have been developed, these may be ineffective if they have MOAs that are similar to those of fungicides to which $B$. cinerea is already resistant; i.e., there may be cross-resistance between the new fungicides and the previously used fungicides. It is, therefore, important to develop a high-throughput screening method to identify fungicide MOAs. A fast exploration of MOAs is helpful for the scientific application of new fungicides.

A series of research methods have been used to reveal fungicide MOAs. The MOA of flumorph was explored by analyzing alterations of hyphal morphology, cell wall

(c) The Author(s). 2019 Open Access This article is distributed under the terms of the Creative Commons Attribution 4.0 International License (http://creativecommons.org/licenses/by/4.0/), which permits unrestricted use, distribution, and 
deposition patterns, F-actin organization, and other organelles in Phytophthora melonis. Results showed that flumorph may be involved in the impairment of cell polar growth through directly or indirectly disrupting the organization of F-actin [6]. Deuterium-labelling was used to determine that the MOA of metalaxyl involved the inhibition of RNA polymerase I [7]. The researcher found that RNA synthesis of phenylamide-sensitive strains, measured as $[3 \mathrm{H}]$ uridine incorporation, was inhibited by about $80 \%$ (Phytophthora megasperma f. $s p$. medicaginis) and by about 40\% (Phytophthora infestans) by metalaxyl and oxadixyl at a concentration of $1 \mu \mathrm{g} / \mathrm{ml}$. RNA synthesis of resistant strains was completely insensitive to metalaxyl and oxadixyl at concentrations as high as $200 \mu \mathrm{g} / \mathrm{ml}$. Additionally, endogenous nuclear RNA polymerase activity of both Phytophthora sensitive isolates appeared to be more sensitive to the phenylamides than of both Phytophthora resistant isolates. These means of cross-resistance could be applied in the bioassay method to determine the MOA by assessing the resistance mechanism. A complex II analysis of mutants of several organisms resistant to succinate dehydrogenase inhibitors (SDHIs), such as carboxin, provided insights into the MOA of SDH-inhibitors $[8$, 9]. Comparison of the sequence from a carboxinsensitive Ustilago maydis strain of iron-sulphur protein (Ip) subunit of succinate dehydrogenase (Sdh) with that of the Ip allele from a carboxin -resistant strain revealed a two-base difference between the sequences. This mutation led to the substitution of a leucine residue for a histidine residue within the third cysteine-rich cluster of the deduced amino-acid sequence of the Ip allele. This cluster, which is associated with the S3 iron-redox centre, is involved in the transport of electrons from succinate to ubiquinone $(\mathrm{Q})$. Confirmation that this nucleotide change led to enhanced resistance to carboxin was obtained following mutagenesis of the sensitive Ip allele to the resistant form and expression of the mutated allele in $U$. maydis [8]. A patent proposed the use of affinity chromatography to determine the MOA of oxathiapiprolin [10]. The authors found that the oxathiapiprolin specifically binds to Oomycete oxysterol binding polypeptide in the total protein mixture obtained from Oomycete. The MOA of quinone outside Inhibitors (QoI) was explored using protein crystallization combined with molecular docking. The existence of more than 40 different fungicide MOAs (FRAC, 2019) makes screening by the methods above time-consuming and costly. Thus, fast, robust, and high-throughput screening techniques are required.

In recent years, the application of omics approaches has greatly accelerated the progress of MOA identification for new fungicides. For example, the MOA of phenamacril against Fusarium graminearum was studied by the high-throughput sequencing of the fungal genome and transcriptome [11], and proteomics was applied to study the MOA of pyrimorph [12]. To determine the mechanism of resistance to the fungicide phenamacril in $F$. graminearum, the researchers sequenced and annotated the genome of the resistant strain YP-1. They sequenced 22 functional annotated genes of another $F$. graminearum sensitive strain (strain 2021) and corresponding resistant strains. The only mutation common to all of the resistant mutants occurred in the gene encoding myosin-5 (point mutations at codon 216, 217, 418,420 , or 786 ). Further, they found that transformed mutants with a copy of the resistant myosin- 5 locus fragment exhibited resistance to phenamacril, and the transformed mutant with a copy of the sensitive fragment exhibited sensitivity to phenamacril [11]. The proteomic response of Phytophthora capsici to pyrimorph was investigated using the iTRAQ technology to determine the target site of the fungicide and potential biomarker candidates of drug efficacy. Many of the proteins with altered expression were associated with glucose and energy metabolism. Biochemical analysis using D-[U-14C] glucose verified the proteomics data, suggesting that the major MOA of pyrimorph on P. capsici is the inhibition of cell wall biosynthesis [12]. Metabolomics involves the comprehensive qualitative and quantitative profiling of a large number of metabolites of a biological system. The major advantage is the simultaneous monitoring of metabolic networks, which enables their changes to be associated with biotic and/or abiotic causal agents and enables the detection of corresponding biomarkers. The metabolic footprinting of Saccharomyces cerevisiae provided sufficient information to discern the MOAs of antifungal substances. Respiratory inhibitors were discriminated from other kinds of inhibitors, while relationships between metabolites and MOAs were not described in detail [13]. The use of metabolomics for the study of fungicides MOAs is still in its infancy [14]. Thus, metabolomics is a powerful tool to identify the MOAs of antifungal and antibiotic compounds by fingerprinting model discrimination, biomarker identification, and metabolic pathway excavation.

In this paper, the effects of different fungicide MOAs on the metabolome of $B$. cinerea were studied. A metabolic fingerprint model was established, the use of biomarkers was investigated, and the MOA of a novel fungicide, SYP-14288, was predicted.

\section{Results}

Sensitivity of $B$. cinerea to fungicides

The $\mathrm{EC}_{50}$ values of the 14 fungicides tested against SP2-6 are shown in Table 1 . They ranged from $0.004 \mu \mathrm{g} / \mathrm{mL}$ to $5.41 \mu \mathrm{g} / \mathrm{mL}$ which indicated that SP2-6 has a good sensitivity to the fungicides. Among the fungicides tested, 
fluazinam and SYP-14288 showed the greatest inhibitory activities, with $\mathrm{EC}_{50}$ values lower than $0.01 \mu \mathrm{g} / \mathrm{mL}$.

\section{Metabolome of $B$. cinerea}

The $B$. cinerea mycelia was grown on PDA plates supplemented individually with 14 fungicides $\left(E_{50}\right)$ and DMSO $(0.1 \% \mathrm{v} / \mathrm{v}$, control) for 3 days. After incubation, the metabolites of the homogenized mycelia were extracted, dried and derivatized by methoximation and trimethylsilylation prior to GC-MS analysis. A typical total ion current chromatogram is shown in Fig. 1. The detected B. cinerea metabolome consisted of 245 metabolites based on NIST 2005, including mainly amino acids, organic acids, alcohols, sugars, alkanes, and organic esters. The relative standard deviation (RSD) of the internal standard substance salicin was $3.49 \%$, which indicated the acceptable stability of the instrument. The RSDs of the metabolites in the 6 technical replicates ranged from 3.53 to $245 \%$, which was in accordance with a previous report [15].

\section{HCA of the metabolic fingerprint of $B$. cinerea treated with fungicides having different MOAs}

The HCA was dominated by six distinct groups that were very similar to groups based on MOAs in FRAC (Fig. 2, Additional file 1). One group contained the methionine biosynthesis fungicides pyrimethanil and cyprodinil, multi site action inhibitor thiram. The second group contained the fungicides imazalil and fenhexamid that interfered with sterol biosynthesis. The third group contained the respiration inhibitors fungicides boscalid and kresoxim-methyl, multi site action inhibitor clorothalonil. The fourth group contained the fungicides fludioxonil and procymidone that interfered

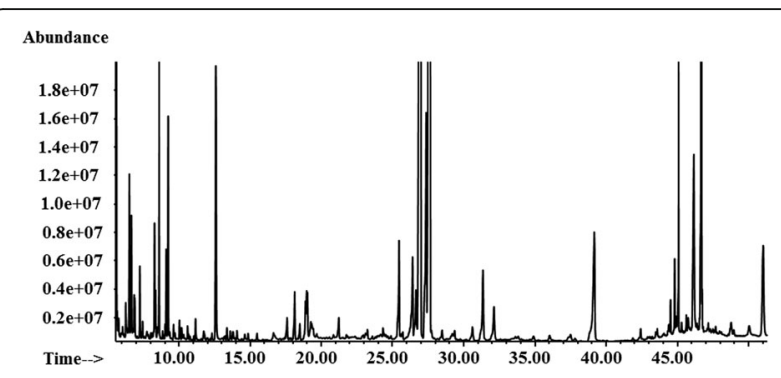

Fig. 1 Total ion current chromatogram from a typical analysis of the metabolome of B. cinerea SP2-6. Abundance means the amount of ion. Time means retention time / min

with signal transduction. The fifth group contained the fungicides carbendazim and thiophanatemethyl that act during $\beta$-tubulins assembly. The last group contained the uncoupler of oxidative phosphorylation fluazinam and the MOA unknown fungicide SYP-14288.

\section{The $B$. cinerea metabolome fluctuates after exposure to fungicides having different MOAs}

The concentration and composition of metabolites were compared between the fungicide treatment and control groups using the one-way ANOVA followed by a Tukey's test at a significance level of $p<0.05$. In response to treatment with different chemicals, the metabolome of $B$. cinerea fluctuated, the number of detected metabolites ranging from 11 to 83 . Table 2 shows the amount of metabolites specifically and commonly up-regulated and down-regulated after treatment with different fungicides within the same MOA group (Additional file 2).

The results that fungicides of the same MOA share a large number of commonly changed metabolites

Table $1 \mathrm{EC}_{50}$ values of different mode of action fungicides to B. cinerea SP2-6

\begin{tabular}{|c|c|c|c|c|}
\hline Mode of action group & Fungicides & CAS & $\mathrm{EC}_{50}(\mu \mathrm{g} / \mathrm{mL})$ & Concentrations used $(\mu \mathrm{g} / \mathrm{mL})$ \\
\hline \multirow[t]{2}{*}{$\beta$-tubulins assembly } & Carbendazim & $10,605-21-7$ & 0.04 & $0,0.005,0.013,0.05,0.1,0.2$ \\
\hline & Thiophanatemethyl & $23,564-05-8$ & 0.36 & $0,0.2,0.3,0.4,0.6,0.9$ \\
\hline \multirow[t]{3}{*}{ Respiration } & Boscalid & $188,425-85-6$ & 0.08 & $0,0.02,0.05,0.1,0.5,1$ \\
\hline & Kresoxim-methyl & $143,390-89-0$ & 0.13 & $0,0.02,0.04,0.08,0.78,1.56$ \\
\hline & Fluazinam & $79,622-59-6$ & 0.01 & $0,0.001,0.005,0.05,0.1,0.2$ \\
\hline \multirow[t]{2}{*}{ Mitochondrial function } & Cyprodinil & $121,552-61-2$ & 0.47 & $0,0.15,0.3,1,3,15$ \\
\hline & Pyrimethanil & $53,112-28-0$ & 0.68 & $0,0.01,0.5,1,2,5$ \\
\hline \multirow[t]{2}{*}{ Signal transduction } & Fludioxonil & $131,341-86-1$ & 0.02 & $0,0.005,0.01,0.02,0.04,0.05$ \\
\hline & Procymidone & $32,809-16-8$ & 0.39 & $0,0.23,0.45,0.6,0.8,0.9$ \\
\hline \multirow[t]{2}{*}{ Membrane sterol biosynthesis } & Imazalil & $35,554-44-0$ & 4.64 & $0,1,2.5,5,10,15$ \\
\hline & Fenhexamid & $126,833-17-8$ & 0.41 & $0,0.1,0.25,0.5,1,5$ \\
\hline \multirow[t]{2}{*}{ Multi site action } & Chlorothalonil & $1897-45-6$ & 5.41 & $0,0.1,0.5,1,10,50$ \\
\hline & Thiram & $137-26-8$ & 1.59 & $0,0.39,0.78,1.56,3.13,6.25$ \\
\hline Unknown & SYP-14288 & & 0.004 & $0,0.001,0.003,0.005,0.01,0.05$ \\
\hline
\end{tabular}

Note: The $\mathrm{EC}_{50}$ values of fungicides against $B$. cinerea SP2- 6 were measured based on the mycelial growth rate method on PDA plates. Each combination of isolate and fungicide concentration was represented by 6 replicate plates, and the experiments were performed 3 times 


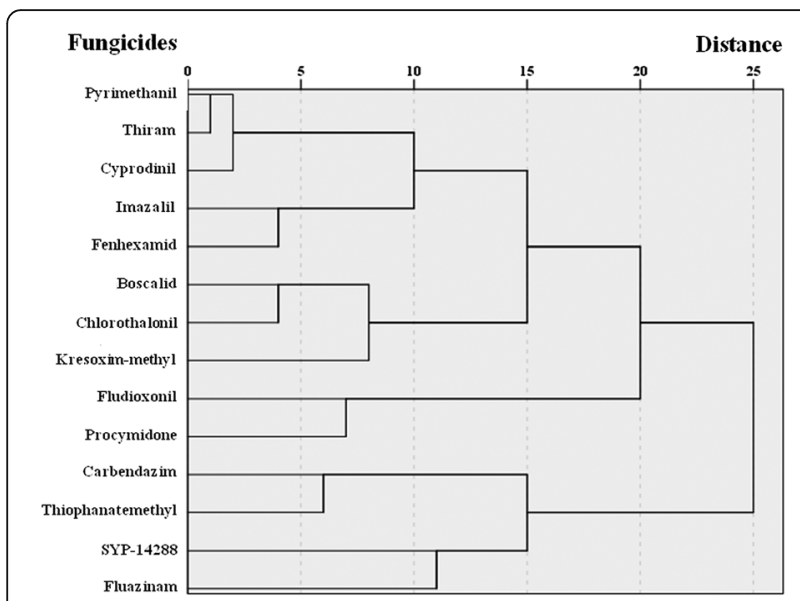

Fig. 2 Clustering analysis results of the studied fungicides by metabolic fingerprinting. 179 metabolites of 6 replicates went into the clustering analysis. Based on metabolites and their relative contents of $B$. cinerea SP2-6, most of the 14 fungicides were discriminated as 6 groups which coincided with their modes of actions in FRAC

indicated that they disturbed similar metabolic pathways. In this research, the two fungicides affecting mitochondrial processes [16] and amino acid biosynthesis, cyprodinil and pyrimethanil, induced down-regulation of 16 identical metabolites and up-regulation of 1 identical metabolites, and the two fungicides affecting signal transduction related to osmoregulation, procymidone and fludioxonil, induced up-regulation of 15 and downregulation of 10 identical metabolites.
At the same time, the existence of the specifically changed metabolites of $B$. cinerea by each fungicide may be related to their belonging to different MOA subgroup and the difference in chemical structures. For instance, boscalid, kresoxim-methyl and fluazinam, belonging to three subgroups as inhibition of complex II, complex III cytochrome bc1(ubiquinol oxidase) at Qo site and uncouplers of oxidative phosphorylation, induced 27, 26 and 66 specifically changed metabolites in B. cinerea. For carbendazim and thiophanatemethyl, although they are both from the same subgroup as $\beta$-tubulin inhibitors, the chemical group of the latter has been reported to act with toxicity only after metabolism in organism [17]. This may be a reason to explain the specifically changed metabolites difference as 50 and 24 induced by the two fungicides.

\section{Biomarker analysis of succinate dehydrogenase-inhibitors and methionine biosynthesis-inhibitor}

In this study, succinate content specifically increased both in boscalid and thiram treated mycelium. In contrast, succinate levels were downregulated after treatment with cyprodinil, pyrimethanil and fludioxonil, or they remained unaffected in the presence of other fungicides. More than 40 times of upregulation was detected in the mycelia of SP2-6 treated by boscalid (Fig. 3a, b).

The one-way ANOVA (followed by a Tukey's test at a significance level of $p<0.05$ ) of metabolites in SP2-6 treated with or without methionine biosynthesis inhibitors showed that the total amount of cystathionine increased significantly. It increased 1.1 and 1.5 times in

Table 2 Changes in SP2-6 metabolites after exposure to different fungicides

\begin{tabular}{|c|c|c|c|}
\hline \multirow[t]{2}{*}{ Mode of action group } & \multirow[t]{2}{*}{ Fungicides } & \multicolumn{2}{|c|}{ Number of metabolites changed (up, down) } \\
\hline & & Specifically changed & Commonly changed \\
\hline \multirow[t]{2}{*}{$\beta$-tubulins assembly } & Carbendazim & $50(48,2)$ & $8(7,1)$ \\
\hline & Thiophanatemethyl & $24(16,8)$ & \\
\hline \multirow[t]{3}{*}{ Respiration } & Boscalid & $27(15,12)$ & $11(6,5)$ \\
\hline & Kresoxim-methyl & $26(22,4)$ & \\
\hline & Fluazinam & $66(62,4)$ & \\
\hline \multirow[t]{2}{*}{ Mitochondrial function } & Cyprodinil & $4(1,3)$ & $17(1,16)$ \\
\hline & Pyrimethanil & $8(1,7)$ & \\
\hline \multirow[t]{2}{*}{ Signal transduction } & Procymidone & $22(21,1)$ & $25(15,10)$ \\
\hline & Fludioxonil & $55(44,11)$ & \\
\hline \multirow[t]{2}{*}{ Membrane sterol biosynthesis } & Fenhexamid & $14(10,4)$ & $7(2,5)$ \\
\hline & Imazalil & $4(2,2)$ & \\
\hline \multirow[t]{2}{*}{ Multi site action } & Chlorothalonil & $83(65,18)$ & n.a. \\
\hline & Thiram & $37(15,22)$ & n.a. \\
\hline Unknown & SYP-14288 & $20(15,5)$ & n.a. \\
\hline
\end{tabular}

Note: The concentration and composition of metabolites were compared between the fungicide treatment and control groups using the one-way ANOVA followed by a Tukey's test at a significance level of $p<0.05$. Metabolites changed column showed the number of metabolites specifically (fungicide specific unique) and commonly regulated within the same mode of action group after treated by different fungicides. "n.a." means not analysed 
the mycelia of SP2-6 after pyrimethanil and cyprodinil treatments, respectively (Fig. 4). Treatments with other fungicides did not lead to significant changes in cystathionine contents.

\section{The preliminary prediction MOA of SYP-14288}

In addition, the ANOVA analysis showed that SYP-14288 induced metabolite changed were similar to those caused by fluazinam. Among the 20 metabolites with significant differences after treatment with SYP-14288, 18 were common to fluazinam treatments. The contents of 15 metabolites were increased, while the other 3 were reduced.

\section{Discussion}

\section{Sensitivity of $B$. cinerea to fungicides}

A sensitive strain is necessary to explore the MOAs of fungicides. The compounds commonly combine with their target proteins effectively and inhibit the enzymes' activities. Here, we selected the strain SP2-6, which is susceptible to the MOAs of typical fungicides used for the control of grey mold in the field, such as carbendazim, and pyrimethanil. We inferred the level of susceptibility according to the MIC of fungicides. In addition, the strain is presumed to be sensitive to fungicides of the same MOA when sensitive to a certain fungicide.

\section{Discrimination of MOAs by HCA}

The HCA of the metabolic profile of $B$. cinerea after fungicides treatments classified most of the test fungicides into groups that showed good agreement with the six types of MOAs. Thus, the metabolites of $B$. cinerea may reflect the characteristics of fungicides with similar MOAs.

The relationships between multi-site fungicides and respiration inhibitors or methionine biosynthesis inhibitors were close. Multi-site fungicides caused a wider range of metabolic differences and, to some extents they

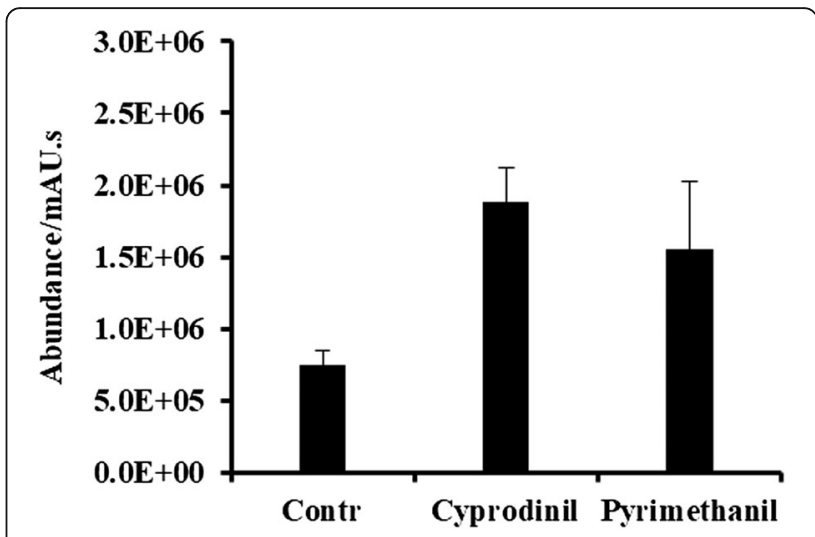

Fig. 4 Amount of cystathionine in B. cinerea mycelium after treatment with methionine biosynthesis-inhibitor. Contr is the control value. Each value is the mean after 6 replicates \pm standard deviation. Abundance/mAU.s means the unit of peak area

had similar metabolic differences compared with respiration inhibitors and methionine biosynthesis inhibitors.

In this study, the metabolic fingerprints of the SP2-6 mycelia grown on PDA plates supplemented with fungicides having similar targets clustered together. The clustering analysis of the metabolic fingerprinting reflected the MOAs of the tested fungicides. We can assume that HCA clustering can be performed using the above system when speculating on the MOA of fungicides. Fungicides with unknown MOA will be aggregated with ones of similar MOA to provide an effective reference for our prediction. Thus, the metabolic fingerprints of fungicides have the potential to be used to group fungicides based on their MOAs.

The results are consistent with a report [13] on the separation of non-respiratory inhibitors from respiratory inhibitors, and complex II and III respiratory inhibitors from sterol biosynthesis inhibitors. However, $\beta$-tubulin assembly inhibitors and signal transduction inhibitors

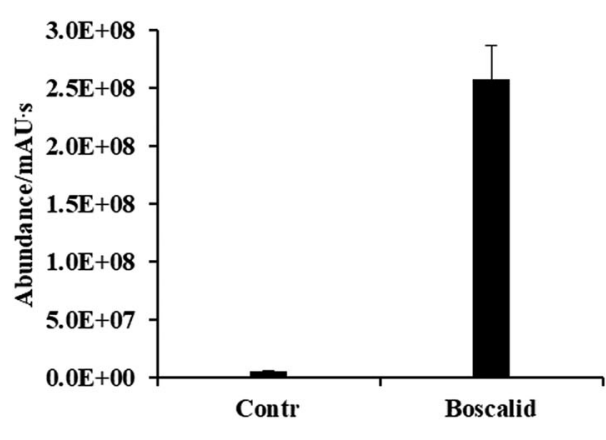

a

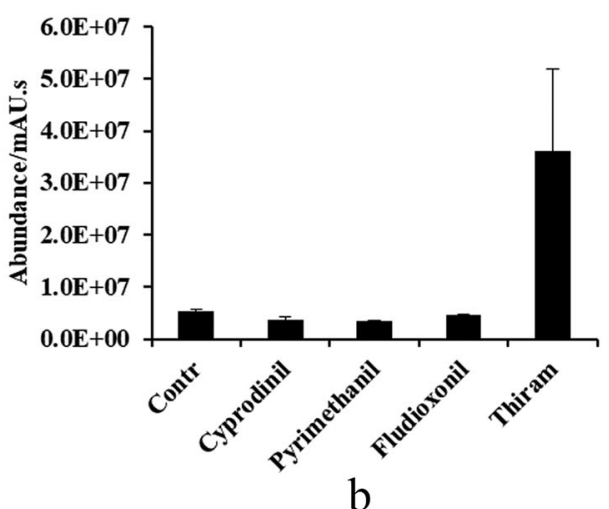

Fig. 3 Amount of succinate in B. cinerea mycelium after treatment with succinate dehydrogenase-inhibitors (a) and other inhibitors (b). Contr is the control value. Each value is the mean after 6 replicates \pm standard deviation. Abundance/mAU.s means the unit of peak area 
did not follow the rule. The uncoupler formed a separate class in this study, which is different from the literature in which fluazinam and sterol biosynthesis inhibitors form a group. However, fluazinam is distant from the other respiratory inhibitors and this is related to its MOA being different from those of the other inhibitors.

\section{Biomarkers of fungicide MOAs}

Metabolites that vary significantly in the fungicide treatment group may be directly or indirectly related to the mechanism of action, and some specific metabolites of which can be identified as potential biomarkers of certain fungicide MOAs.

\section{Succinate dehydrogenase inhibitors}

In this study, the content of succinate increased more than 40 times in the mycelia of SP2-6 after the boscalid treatment. This accumulation is in accordance with the mechanism. Boscalid is a succinate dehydrogenase inhibitor that targets the succinate dehydrogenase complex in the respiratory chain [18]. This enzyme complex couples the oxidation of succinate to fumarate in the mitochondrial matrix (or in the cytoplasmic membrane of pathogens) with the reduction of ubiquinone to ubiquinol in the membrane during aerobic respiration [19]. The fungicide interferes with the succinate dehydrogenase complex to block the oxidation of succinate to fumarate and affects the normal life activities of pathogens. It is speculated that the succinate content increased in the mycelia of SP2-6 after the multi-site action inhibitors thiram treatment related to the mechanism of it acting on the electron transport chain. However, the succinate content decreased significantly after cyprodinil, pyrimethanil and fludioxonil treatment. Thus, succinate can be a good biomarker of succinate dehydrogenase inhibitors.

\section{Methionine biosynthesis inhibitors}

In this study, the total amount of cystathionine increased significantly in metabolites of SP2-6 treated with pyrimethanil and cyprodinil. This was consistent with previous research that pyrimethanil and cyprodinil may be disturbing the action of cystathionine $\beta$-synthase on synthesis of methionine from cystathionine [4]. However, another research has showed that anilinopyrimidine primarily target the mitochondria. Thus, the role of cystathionine as a biomarker identified by metabolomics remains to be further verified.

\section{Predicting SYP-14288's MOA}

SYP-14288 and fluazinam treatment induced similar regulation in metabolic level, SYP-14288, belonging to the diarylamines, has a structure similar to that of fluazinam [20]. It is suggested that the MOA of SYP-14288 is similar to that of fluazinam, which act as an uncoupler of oxidative phosphorylation [21].

SYP-14288 and fluazinam were clustered into one group (Fig. 2), indicating that they share the same MOA. We can safely predict that SYP-14288 is an uncoupler of oxidative phosphorylation since the MOA recognition through clustering has been reported several times in medical research. Metabolic profiles of Staphylococcus aureus after treatments with antibiotics having known MOAs have been compared with those obtained from treatments with the antibiotic compounds being tested. Using this platform, $\mathrm{Yu}$ et al. [22] performed a principal components analysis (PCA) and revealed that the antibacterial MOAs of rhizome extracts of the plant Tinospora capillipes Gagnep. resembled those of rifampicin and norfloxacin, which act on nucleic acids. Similarly, Yi et al. [23] performed a PCA and found that the MOA of berberine may be similar to that of rifampicin or norfloxacin. Also using $S$. aureus as the model organism, Liu et al. [24] performed GC-MS metabolomics in combination with HCA and concluded that the MOAs of the four synthesized antibiotics under study resembled that of clindamycin, which inhibits protein synthesis by reversibly binding to the $50 \mathrm{~S}$ subunit of ribosomes. Thus, the MOAs of new agents can be explored based on metabolome and cluster analyses.

We have evidence which makes it plausible that at least one MOA of SYP-14288 may be an uncoupler of oxidative phosphorylation. However, we still need to further verify and analyze the metabolic pathways affected by SYP-14288 and related enzymes.

\section{Conclusions}

Here, we described a comprehensively and quickly exploring approach for determining the MOAs of unknown antifungals using the microbial metabolic fingerprinting models for fungicides with known MOAs. First, we tested this hypothesis in a $B$. cinerea model, using mycelia as the target and 13 fungicides as exogenous metabolic challenges. Second, we analyzed fungicides having the same MOAs by HCA or by comparing normal mycelia to mycelia that were exposed to a sub-lethal dose of SYP-14288 to identify biomarkers. Theoretically, this metabolomics methodology could be extended (1) to predict tentative MOAs for new fungicides, and (2) to identify the disturbed metabolic pathways.

The HCA was performed based on the B. cinerea metabolome and these 13 fungicides correctly clustered in to 6 different MOA groups. The biomarkers for succinate dehydrogenase and methionine synthesis inhibitors were obtained using a one-way ANOVA between treatments and control metabolites. Not only did SYP-14288 clustered near fluazinam, but also a large number of similar 
metabolome changes occurred in both of them, indicating that these two fungicides may have a similar MOA.

The metabolome fluctuations mirrored the changes downstream of the pathogen under disturbance, but the targets of the fungicides need to be determined using molecular biology and biochemistry. Compared with the control group, the metabolic fingerprint of SP2-6 changed after being exposed to fungicides. The metabolic fingerprints differed among different fungicide treatments. These altered metabolites were correlated with the fungicides' MOAs and have the potential to be used as MOA biomarkers. In addition, fungicides having similar MOAs had short clustering distances and the changes in the metabolic fingerprint of SP2-6 can reflect the MOAs. Thus, metabolic fingerprinting can be a promising approach to quickly gain insight in to the potential MOAs of fungicides, which then can direct the follow up validation work using molecular genetic tools.

\section{Methods}

\section{Chemicals and materials}

The 14 fungicides (with known and unknown MOAs) included the $\beta$-tubulin assembly inhibitors carbendazim (Sichuan Guoguang Agrochemicals Co., Ltd) and thiophanatemethyl (Hainan Li Zhi Biological Co., Ltd.), the respiration inhibitors complex II succinate dehydrogenase inhibitor boscalid (Beijing Tak Lida Agricultural Science and Technology Co., Ltd), quinone outside inhibitor kresoxim-methyl (Shandong Jingbo agricultural companies), the uncoupler of oxidative phosphorylation fluazinam (Jiangsu Youshi Chemical Co., Ltd), the methionine biosynthesis inhibitors cyprodinil (Beijing Mingde Rieter Agriculture Branch Co., Ltd) and pyrimethanil (Tianjin Shi Pu Le Pesticide Technology Development Co., Ltd), the signal transduction inhibitors fludioxonil (Shandong Weifang Runfeng Chemical Co., Ltd), and procymidone (Lier chemical co., Ltd), the membrane sterol biosynthesis inhibitors imazalil (Liuzhou Huinong Chemical Co., Ltd) and fenhexamid (Ai Li Sida Biological Chemicals Co., Ltd), and the multi-site action inhibitors chlorothalonil (Jiangyin Suli Chemical Co., Ltd) and thiram (Yingkou Leike Pesticide Co., Ltd.) (Table 1). The fungicides were from commercial sources with a minimum purity of $95 \%$. SYP-14288, a new fungicide with an unknown MOA, which was created by imitating fluazinam, came from the Shenyang Chemical Industry Co. Ltd. The fungicides were dissolved in dimethyl sulfoxide (DMSO; Xilong Chemical Co., Ltd.) to produce $10^{4} \mu \mathrm{g} / \mathrm{mL}$ stock solutions. From these stocks, other additional stock concentrations were prepared by serial dilution. Stocks were aliquoted into $2 \mathrm{~mL}$ sterile microcentrifuge tubes and stored at $-4{ }^{\circ} \mathrm{C}$ in the dark.

Methanol (chromatographically pure) was purchased from the Sinopharm Chemical Reagent Co., Ltd. Ultrapure water was obtained from a milli-Q system (Millipore). $\mathrm{N}, \mathrm{O}$-Bis (trimethylsilyl) trifluoroacetamide $(\mathrm{v} / \mathrm{v}$; contains $1 \%$ trimethylchlorosilane (TMCS), 99\%), pyridine (99.8\%), methoxyamine hydrochloride (98\%), internal standard salicin and 40 other metabolite standards with active ingredients greater than $99 \%$ were purchased from Sigma-Aldrich.

\section{Sensitive strain selection and $\mathrm{EC}_{50}$ measurements}

$B$. cinerea strain SP2-6, which is sensitive to 6 fungicides MOAs, was selected from 115 isolates taken from tomato plants suffering grey mold in 8 Chinese provinces from 2006 to 2015 . The isolates were identified as $B$. cinerea based on morphology and the DNA sequence data of three nuclear protein-coding genes glyceraldehyde-3phosphate dehydrogenase (G3PDH), Heat-shock Protein 60 (HSP60), and DNA-dependent RNA polymerase subunit II (RPB2) [25]. First, the sensitive B. cinerea strain were selected by observing the failure to grow on PDA plates independently supplemented with the minimum inhibitory concentration (MIC) of carbendazim, thiophanatemethyl, boscalid, cyprodinil, procymidone, fludioxonil, and fenhexamid [2628]. Then, $\mathrm{EC}_{50}$ values of kresoxim-methyl, imazalil, and pyrimethanil were tested and then sensitivity baselines [29] were used to screen for sensitive strains. There are limited resistance reports for fluazinam and the multi-site fungicides chlorothalonil and thiram, therefore, no sensitivity determinations were conducted.

The $\mathrm{EC}_{50}$ values of fungicides against strain SP2-6 were measured based on the mycelial growth rate method on PDA plates [30]. The stock solutions were added to molten PDA media when they cooled to approximately $50^{\circ} \mathrm{C}$. For kresoxim-methyl, sensitivity tests were conducted in the presence of $100 \mu \mathrm{g} / \mathrm{mL}$ salicylhydroxamic acid, a specific inhibitor of alternative oxidases.

The strain SP2-6 was grown on PDA plates for 4 days at $20^{\circ} \mathrm{C}$ in the dark before the mycelial plugs were transformed from the colony margin to the center of PDA plates independently supplemented with a serial dilution of a fungicide. To assist their dissolving, DMSO was used at a final concentration of $0.1 \%$ in the medium. Each combination of isolate and fungicide concentration was represented by 6 replicate plates, and the experiments were performed 3 times. After 4 days of cultivation, the radial growth was recorded to calculate the percentage of growth inhibition (relative to the growth on plates without fungicides). $\mathrm{EC}_{50}$ values were calculated by the regression of the probit of the percentage of inhibition of radial growth against the logarithmic values of fungicide concentrations.

\section{Mycelial sample collection}

Mycelial samples of the strain SP2-6 were collected after growing on plates containing the 14 fungicides or DMSO (control). The experimental procedure was as follows: The strain SP2-6 was grown on PDA plates for 
3 days at $20^{\circ} \mathrm{C}$ in the dark. A mycelial plug was taken from the colony margin with a cork borer $(5 \mathrm{~mm})$ and placed mycelial-side down in the center of a PDA plate amended with the $\mathrm{EC}_{50}$ of a fungicide and covered by cellophane (diameter of $4.2 \mathrm{~cm}$ ). For control groups, the mycelia were cultured on PDA plates with a $0.1 \%$ final concentration of DMSO. Each combination of isolate and fungicide concentration was represented by 6 replicate plates, and the experiments were performed three times. After 3 days at $20^{\circ} \mathrm{C}$, all of the mycelia except the plug on the cellophane were harvested with scalpels into $2 \mathrm{~mL}$ centrifuge tubes, frozen with liquid nitrogen, and stored at $-80^{\circ} \mathrm{C}$. At the time of sampling, the mycelia cultivated on plates supplemented with fungicides had almost extended to the margin of the cellophane, while the mycelia cultivated on plates not supplemented with a fungicide (DMSO only) had already grown over the cellophane margin.

\section{Metabolite extraction and derivatization Extraction}

The free polar metabolites were extracted in the supernatants of water-methanol solutions of the homogenized biological samples according to the literatures [31, 32] with some change as follows.

The mycelial samples stored at $-80^{\circ} \mathrm{C}$ were homogenized by ball milling at 30 times/s for 2 min after soaked in liquid nitrogen. The sample aliquots were mixed with $1.8 \mathrm{~mL}$ of solvent mixture (methanol/water, $80 / 20, \mathrm{v} / \mathrm{v}$ ) containing internal standard salicin at $10 \mu \mathrm{g} / \mathrm{mL}$, stored at least for $30 \mathrm{~min}$ on ice), vortexed vigorously for $1 \mathrm{~min}$ and then placed in an ultrasound water bath held at $25^{\circ} \mathrm{C}$ (velocity of ultrasound in water $(1500 \mathrm{~m} / \mathrm{s}$ )/frequency $(40 \mathrm{kHz})=3.75 \mathrm{~cm})$ for $15 \mathrm{~min}$. The samples were centrifuged at $14000 \mathrm{~g}$ for $15 \mathrm{~min}$. Then, $0.6 \mathrm{~mL}$ of supernatant solution was dried in a vacuum centrifuge at $45^{\circ} \mathrm{C}$. The acquired dried metabolite mixture was then used for further analyses.

\section{Derivatization}

For derivatization, $100 \mu \mathrm{L}$ of methoxylamine hydrochloride in pyridine $(20 \mathrm{mg} / \mathrm{mL})$ was added to the dried extractions prior to incubation at $30{ }^{\circ} \mathrm{C}$ for $2 \mathrm{~h}$. Then, $100 \mu \mathrm{L}$ of $\mathrm{N}$, O-bis (trimethylsilyl) trifluoroacetamide containing $1 \%$ trimethylchlorosilane $(\mathrm{v} / \mathrm{v})$ was added, and the mixtures were incubated at $37^{\circ} \mathrm{C}$ for $6 \mathrm{~h}$. After centrifugation at $14000 \mathrm{~g}$ for $15 \mathrm{~min}, 160 \mu \mathrm{L}$ of supernatant was transferred to vials for detection. All of the solutions were tested within $48 \mathrm{~h}$.

\section{GC-MS}

The GC-MS analysis was performed on a QP 2010 GC (Agilent Technologies $6890 \mathrm{~N}$ )-MS (Agilent Technologies $5973 \mathrm{i})$ system using a HP-5MS $(30 \mathrm{~m} \times 0.25 \mathrm{~mm} \times$ $0.25 \mu \mathrm{m}$ ) capillary column. The injector temperature was set to $280^{\circ} \mathrm{C}$ with a split ratio of 5 . Helium was employed as carrier gas at $1 \mathrm{~mL} / \mathrm{min}$. The column temperature was initially kept at $65^{\circ} \mathrm{C}$ for $2 \mathrm{~min}$. It was then increased to $185^{\circ} \mathrm{C}$ by $5^{\circ} \mathrm{C} \mathrm{min}^{-1}$, increased to $200^{\circ} \mathrm{C}$ by $1^{\circ} \mathrm{C} \mathrm{min}^{-1}$, and increased to $280^{\circ} \mathrm{C}$ by $15^{\circ} \mathrm{C}$ $\mathrm{min}^{-1}$, where it was held for $25 \mathrm{~min}$. The MS scan parameters were set as follows: a mass scan range of $\mathrm{m} / \mathrm{z}$ 20-650, a scan interval of $0.2 \mathrm{~s}$, and a detector voltage of $0.9 \mathrm{kV}$. The ion source temperature was $230^{\circ} \mathrm{C}$, and the interface temperature was $280^{\circ} \mathrm{C}$. The solvent cut time was $5.5 \mathrm{~min}$. The injection volume was $1 \mu \mathrm{L}$, constant current mode was used, and the MS electron ionization source was $70 \mathrm{eV}$. The GC-MS data (Enhanced Data Analysis MSD ChemStation Build 75, 26-Aug-2003) were analyzed and qualitative and quantitative data on retention times, names, match qualities were exported by the software. The integrator was that of ChemStation. In the MS Signal Integration Parameters, for Edit Integration Events, the Initial Area Reject was 1, Initial Peak Width was 0.020, Shoulder Detection was OFF, and Initial Threshold was 18.0. In the total ion current chromatogram of a typical sample, one characteristic ion and two reference ions were selected as the qualitative ions to edit the information for all of the metabolites. The names and structures of the metabolites were retrieved from the NIST 2005 database and standards. The structures of the metabolites were based on the reaction rules of metabolites with derivatization reagents [33].

\section{Statistical analyses}

The instrument stability was evaluated by analyzing the percentage of RSD of the internal standard substance in the six repeated tests. The IBM SPSS Statistics 21 software was used to analyze metabolites with one-way ANOVA followed by a Tukey's test at a significance level of $p<0.05$.

HCA were carried out with IBM SPSS Statistics 21 software based on average areas calculated from principal ions of all the compounds with varying content. In an agglomerative HCA, each sample begins as a separate cluster and the algorithm proceeds to combine them until all of the samples belong to one cluster. Two parameters need to be considered when performing hierarchical clustering. The first one is a similarity measurement, such as Euclidean distance, Pearson's correlation, or Spearman's rank correlation. The other parameter is a clustering algorithm, such as average linkage (clustering using the central observations), complete linkage (clustering using the farthest pair of observations between the two groups), single linkage (clustering using the closest pair of observations), or Ward's linkage (clustering to minimize the sum of squares of any two clusters). Clustering results are shown as dendrograms. Here, Euclidean distance and Ward's linkage were used. 


\section{Additional files}

Additional file 1: The quantitative data for the HCA. (XLSX $61 \mathrm{~kb}$ )

Additional file 2: The qualitative informations of commonly-induced metabolites in Table 2. (XLSX 29 kb)

\section{Abbreviations}

GC-MS: gas chromatography-mass spectrometry; HCA: hierarchical clustering analysis; Ip: ironsulphur protein; MIC: minimum inhibitory concentration; MOA: mode(s) of action; PCA: principal components analysis; Qol: quinone outside Inhibitors; RSD: relative standard deviation; SDHIs: succinate dehydrogenase inhibitors; TMCS: trimethylchlorosilane

\section{Acknowledgements}

We gratefully acknowledge the assistance of China agricultural university's key laboratory for biological monitoring and green control for the loans of the gas-chromatography mass-spectrometry instruments used in these analyses.

\section{Authors' contributions}

PFL conceived the idea and guided the overall design of the experiments. $\mathrm{ZHH}$ conducted the experiments, ran the GC-MS of the metabolome, analyzed the data, and drafted the manuscript. LL processed the pretreatment. TD analyzed part of the data. PFL and $\mathrm{ZHH}$ helped in responding to peer reviewers' comments. PFL made critical revisions and approved the final version. XLL contributed to article conception. All authors read and approved the final manuscript.

\section{Funding}

This work was funded by the National Natural Science Foundation of China (3177110697 and U160310041). The funders had no role in design of the study, collection, analysis or interpretation of data, or writing of the manuscript.

\section{Availability of data and materials}

All data and materials are available on request for academic use.

\section{Ethics approval and consent to participate}

This article does not contain any studies with human participants or animals performed by any of the authors.

\section{Consent for publication}

Not applicable.

\section{Competing interests}

The authors declare that they have no competing interests.

Received: 21 February 2019 Accepted: 31 May 2019

Published online: 24 June 2019

\section{References}

1. Williamson B, Tudzynski B, Tudzynski P, Van kan JAL. Botrytis cinerea: the cause of grey mould disease. Mol Plant Pathol. 2007:8(5):561-80.

2. Fan F, Hamada MS, Li N, Li GQ, Luo CX. Multiple fungicide resistance in Botrytis cinerea from greenhouse strawberries in Hubei Province, China. Plant Dis. 2017;101(4):601-6.

3. Jiang JH, Ding LS, Michailides TJ, Li HY, Ma ZH. Molecular characterization of field azoxystrobin-resistant isolates of Botrytis cinerea. Pestic Biochem Physiol. 2009;93(2):72-6.

4. Leroux P, Fritz R, Debieu D, Albertini C, Lanen C, Bach J, Gredt M, Chapeland $F$. Mechanisms of resistance to fungicides in field strains of Botrytis cinerea. Pest Manag Sci. 2002;58(9):876-88.

5. Oshima M, Fujimura M, Banno S, Hashimoto C, Motoyama T, Ichiishi A, Yamaguchi I. A point mutation in the two-component histidine kinase BCOS-1 gene confers dicarboximide resistance in field isolates of Botrytis cinerea. Phytopathology. 2002;92(1):75-80.

6. Zhu SS, Liu XL, Liu PF, Li Y, Li JQ, Wang HM, Yuan SK, Si NG. Flumorph is a novel fungicide that disrupts microfilament organization in Phytophthora melonis. Phytopathology. 2007;97(5):643-9.
7. Fisher DJ, Hayes AL. Mode of action of the systemic fungicides furalaxyl, metalaxyl and ofurace. Pestic Sci. 1982:13(3):330-9.

8. Broomfield PE, Hargreaves JA. A single amino-acid change in the ironSulphur protein subunit of succinate dehydrogenase confers resistance to carboxin in Ustilago maydis. Curr Genet. 1992;22(2):117-21.

9. Keon JP, White GA, Hargreaves JA. Isolation, characterization and sequence of a gene conferring resistance to the systemic fungicide carboxin from the maize smut pathogen. Ustilago maydis. Curr Genet. 1991;19(6):475-81.

10. Andreassi JL, Gutteridge S, Pember SO, Sweigard JA. Detection and screening method and materials useful in performance thereof field of the invention. Int patent W013/009971. 2013.

11. Zheng ZT, Hou YP, Cai YQ, Zhang Y, Li YJ, Zhou MG. Whole-genome sequencing reveals that mutations in myosin-5 confer resistance to the fungicide phenamacril in Fusarium graminearum. Sci Rep. 2015;5:8248.

12. Pang ZL, Chen L, Miao JQ, Wang ZW, Bulone V, Liu XL. Proteomic profile of the plant-pathogenic oomycete Phytophthora capsici in response to the fungicide pyrimorph. Proteomics. 2015;15(17):2972-82.

13. Allen J, Davey HM, Broadhurst D, Rowland JJ, Oliver SG, Kell DB. Discrimination of modes of action of antifungal substances by use of metabolic footprinting. Appl Environ Microbiol. 2004;70(10):6157-65.

14. Aliferis KA, Jabaji S. Metabolomics-a robust bioanalytical approach for the discovery of the modes-of-action of pesticides: a review. Pestic Biochem Physiol. 2044;100(2):105-17.

15. Trygg J, Gullberg J, Johansson Al, Jonsson P, Antti H, Marklund SL, Moritz T. Extraction and GC/MS analysis of the human blood plasma metabolome. Anal Chem. 2005;77(24):8086-94

16. Mosbach A, Edel D, Farmer AD, Widdison S, Barchietto T, Dietrich RA, Corran A, Scalliet G. Anilinopyrimidine resistance in Botrytis cinerea is linked to mitochondrial function. Front Microb. 2017:8:2361.

17. Chen H, Liu X, Wang C, Wang Q, Jiang Y, Yin P, Zhu L. Simultaneous determination of thiophanate-methyl and its metabolite carbendazim in tea using isotope dilution ultra performance liquid chromatography-tandem mass spectrometry. J Chromatogr Sci. 2014;52(10):1157

18. Hägerhäll C. Succinate: quinone oxidoreductases: variations on a conserved theme. Biochim Biophys Acta Bioenerg. 1997;1320(2):107-41.

19. Horsefield R, Yankovskaya V, Sexton G, Whittingham W, Shiomi K, Ōmura S, Byrne B, Cecchini G, Iwata S. Structural and computational analysis of the quinone-binding site of complex II (succinate-ubiquinone oxidoreductase) a mechanism of electron transfer and proton conduction during ubiquinone reduction. J Biol Chem. 2006:281(11):7309-16.

20. Wang ZW, Ni XX, Peng Q, Hou YH, Fang Y, Mu WJ, Liu CL, Liu PF, Liu XL. The novel fungicide SYP-14288 acts as an uncoupler against Phytophthora capsici. Pestic Biochem Physiol. 2018

21. Chapeland F, Fritz R, Lanen C, Gredt M, Leroux P. Inheritance and mechanisms of resistance to anilinopyrimidine fungicides in Botrytis cinerea (Botryotinia fuckeliana). Pestic Biochem Physiol. 1999;64(2):85-100.

22. Yu Y, Yi Z, Liang YZ. Main antimicrobial components of Tinospora capillipes, and their mode of action against Staphylococcus aureus. FEBS Lett. 2007;581(22):4179-83.

23. Yi ZB, Yu Y, Liang YZ, Zeng B. Evaluation of the antimicrobial mode of berberine by LC/ESI-MS combined with principal component analysis. J Pharm Biomed Anal. 2007:44(1):301-4.

24. Liu Y, Wen J, Wang YD, Li Y, Xu WJ. Postulating modes of action of compounds with antimicrobial activities through metabolomics analysis. Chromatographia. 2010;71(3-4):253-8

25. Staats M, Baarlen P, Kan JAL. Molecular phylogeny of the plant pathogenic genus Botrytis and the evolution of host specificity. Mol Biol Evol. 2005;22(2):333-46.

26. Yarden O, Katan T. Mutation leading to substitutions at amino acids 198 and 200 of beta-tubulin that correlated with benomyl-resistance phenotypes of field strains of Botrytis cinerea. Phytopathology. 1993;83:1478-83.

27. Ji ZL, Tong YH, Zhang JJ, Xu JY, Chen XJ, Xia H. Resistance of Botrytis cinered to procymidone. Journal of Yangzhou University. 2003:24(3):60-3.

28. Fernández-Ortuño D, Grabke A, Bryson PK, Amiri A, Peres NA. Fungicide resistance profiles in Botrytis cinerea from strawberry fields of seven southern US states. Plant Dis. 2014;98(6):825-33.

29. Zhao H, Kim YK, Huang L, Xiao CL. Resistance to thiabendazole and baseline sensitivity to fludioxonil and pyrimethanil in Botrytis cinerea populations from apple and pear in Washington state. Postharvest Biol Technol. 2010; 56(1):12-8.

30. Yourman LF, Jeffers SN. Resistance to benzimidazole and dicarboximide fungicides in greenhouse isolates of Botrytis cinerea. Plant Dis. 1999;83(6):569-75. 
31. Aliferis KA, Cubeta MA, Jabaji S. Chemotaxonomy of fungi in the Rhizoctonia solani species complex performing GC/MS metabolite profiling. Metabolomics. 2013;9(1):159-69.

32. Warth B, Parich A, Bueschl C, Schoefbeck D, Neumann NKN, Kluger B, Schuster K, Krska R, Adam G, Lemmens M, Schuhmacher R. GC-MS based targeted metabolic profiling identifies changes in the wheat metabolome following deoxynivalenol treatment. Metabolomics. 2015;11(3):722-38.

33. Kanani H, Chrysanthopoulos PK, Klapa MI. Standardizing GC-MS metabolomics. J Chromatogr B Biomed Sci Appl. 2008;871(2):191-201.

\section{Publisher's Note}

Springer Nature remains neutral with regard to jurisdictional claims in published maps and institutional affiliations.

Ready to submit your research? Choose BMC and benefit from:

- fast, convenient online submission

- thorough peer review by experienced researchers in your field

- rapid publication on acceptance

- support for research data, including large and complex data types

- gold Open Access which fosters wider collaboration and increased citations

- maximum visibility for your research: over $100 \mathrm{M}$ website views per year

At $\mathrm{BMC}$, research is always in progress.

Learn more biomedcentral.com/submissions 